\title{
Performance of a Genetic Algorithm for Solving the Multi-Objective, Multimodal Transportation Network Design Problem
}

\author{
Ties Brands and Eric C. van Berkum \\ Centre for Transport Studies, University of Twente \\ Postbus 217, 7500AE Enschede, the Netherlands \\ t.brands@utwente.nl,e.c.vanberkum@utwente.nl
}

\begin{abstract}
The optimization of infrastructure planning in a multimodal network is defined as a multiobjective network design problem, with accessibility, use of urban space by parking, operating deficit and climate impact as objectives. Decision variables are the location of park and ride facilities, train stations and the frequency of public transport lines. For a case study the Pareto set is estimated by the Non-dominated Sorting Genetic Algorithm (NSGA-II). Such a Pareto set is one specific outcome of the optimization process, for a specific value of the parameters generation size, number of generations and mutation rate and for a specific outcome of the Monte Carlo simulation within NSGA-II. Similar issues exist for many other metaheuristics. However, when applied in practice, a policy maker desires a result that is robust for these unknown aspects of the method. In this paper Pareto sets from various runs of the NSGA-II algorithm are analyzed and compared. To compare the values of the decision variables in the Pareto sets, new methods are necessary, so these are defined and applied. The results show that the differences concerning decision variables are considerably larger than the differences concerning objectives. This indicates that the randomness of the algorithm may be a problem when determining the decisions to be made. Furthermore, it is concluded that variations caused by different parameters are comparable with the variations caused by randomness within the algorithm.
\end{abstract}

Keywords: Multi-objective optimization, genetic algorithm, NSGA-II, multimodal passenger transportation networks, Pareto set comparison

\section{Introduction}

Highly urbanized regions in the world nowadays face well-known sustainability problems in traffic systems, like congestion, use of scarce space in cities by vehicles and the emission of greenhouse gases. A shift from car to public transport (PT) modes is likely to alleviate these problems. Extension of the PT network may contribute to this shift by enhancing the quality of PT trips. However, investments in PT infrastructure require large financial resources. Better utilization of the existing PT infrastructure without the need for big investments in large infrastructural developments may be achieved by facilitating an easy transfer from private modes (bicycle and car) to PT modes (bus, tram, metro, train). This transfer can be made easier by network developments that enable multimodal trips, like opening new Park and Ride (P\&R) facilities, opening new train stations or changing existing or opening new PT service lines.

When such transportation network developments are planned, the decision is often based on an evaluation of a few pre-defined scenarios. The composition of the scenarios is usually based on expert judgment, and the assessment is usually based on a multi criteria analysis. 
However, there is a fair chance that the scenario that is selected as the best can still be improved.

For this reason applying an optimization technique is preferred. In this paper, a multiobjective network optimization approach is adopted, which enables us to identify trade-offs between objectives (see also [1]), because the field of transportation planning is, like many other fields, a typical example of a situation that involves more than one objective [2].

Network design problems (NDPs) have received a lot of attention in the literature, in many different versions. One subclass of problems is the transit network design problem, which has been studied in various ways, as reviewed by [3]. This includes greedy algorithms, evolutionary algorithms and design meetings involving expert judgments. Another subclass of problems is the unimodal road network design problem, which has also been studied in various ways [4].

Applications in a multimodal context are less common, but they do exists, for example road link capacity and bus routes [5] or pricing of private and public links [6]. The NDP is considered a multi-objective problem in only a limited number of cases. Examples of objectives being considered simultaneously are travel time and construction costs [7], travel time and travel time variation [8], travel time and $\mathrm{CO}$ emissions $[9,10]$ and social welfare improvement, revenue generation and equity [11]. Finally, [5] simultaneously maximize user benefits, passenger share of the bus mode, service coverage, while minimizing average generalized travel cost for a bus mode trip. To our knowledge [5] is the only paper combining multi-objective optimization with a multimodal NDP, considering both new street construction and lane additions / allocations as well as redesign of bus routes. The focus is on the development of the metaheuristics to solve the problem: the performance of a hybrid genetic algorithm and a hybrid clonal selection algorithm are compared, using multiple test networks. In the lower level, car and bus are distinguished as separate modes. A number of papers applied metaheuristics for solving the transit network design problem, for example [12-14] use a genetic algorithm, [15] uses simulated annealing, [16] tests both scatter search as a genetic algorithm and [17] uses a metaheuristic called GRASP (Greedy Randomized Adaptive Search Procedure).

The randomness in such algorithms is usually addressed by doing multiple runs and presenting average values, for example when comparing various (variants of) solution algorithms (as is done for example in [2]). An aspect that is less addressed is the variation of the results within one variant of a solution algorithm, with respect to the variation of the results between two variants of a solution algorithm. Another aspect is the comparison of decision variables of optimization outcome. To be able to compare various results in the case of multi-objective optimization, one must be able to compare Pareto sets with each other, both concerning objective values as well as values of decision variables.

Firstly, the contribution of this paper is to formalize methods to compare Pareto sets based on the values of decision variables. Secondly, the impact of randomness and parameter settings in the application of a metaheuristic like NSGA-II on the outcome of the optimization process is assessed, for the case of a network design problem with multimodal decision variables. Therefore, existing indicators to compare the objective values of different Pareto sets are used, in addition to the newly developed methods to compare the values of decision variables.

Section 2 defines the problem in more detail and describes the solution method applied. Section 3 defines methods to compare Pareto sets with each other, to be used in Section 4, where the outcomes of a real world case study are analyzed. The case study is described and the values of various outcomes of the optimization process are compared with each other. This leads to conclusions in the final section. 


\section{Problem Definition}

\subsection{Bi-level Problem}

The transportation network design problem is often solved as a bi-level optimization problem, to correctly incorporate the reaction of the transportation system users to network changes, as is argued by [18-20]. In our research, the network design problem is regarded as a bi-level system as well (see Figure 1). The upper level represents the behavior of the network authority, optimizing system objectives. In the lower level the travelers minimize their own generalized costs (e.g., travel time, cost), by making individually optimal choices in the multimodal network, considering variety in travel preferences among travelers. The network design in the upper level interacts with the behavior of the travelers in the network: the lower level. This is put into operation by a transport model, which assumes a stochastic user equilibrium (no driver can unilaterally change routes to improve his/her perceived generalized travel costs). For any network design the planner chooses, the transport model yields a network state (e.g., travel times and loads), from which the values of all objective functions can be derived. The equilibrium in the lower level is a constraint for the upper level problem.

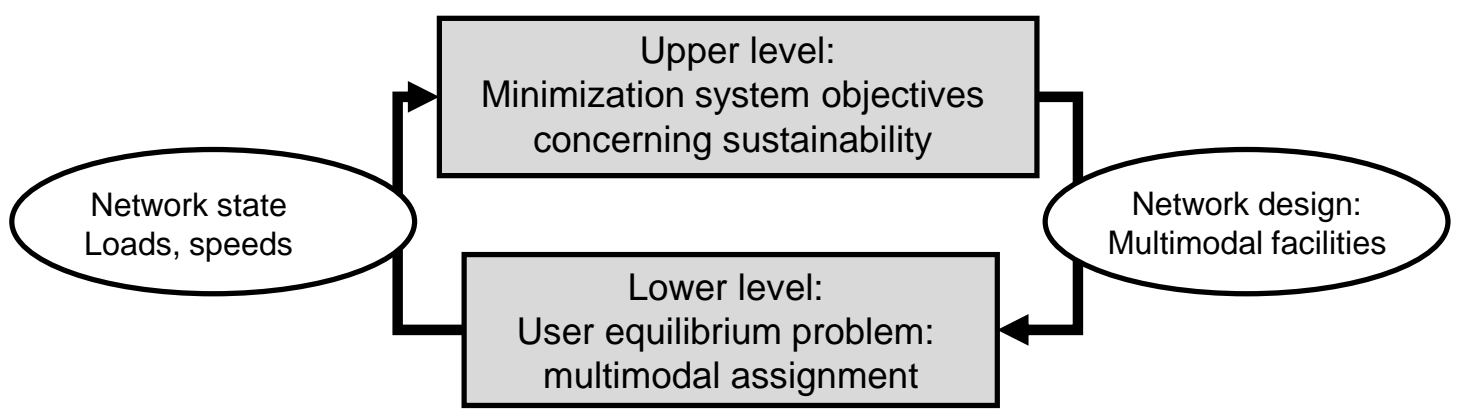

Figure 1. The Bi-level Optimization Problem

\subsection{Network and Demand Definition}

The multimodal transportation network is defined as a directed graph $G$, consisting of node set $N$, link set $A$, a line set $L$ and a stop set $U$ (see Table 1 ). For each link a one or more modes are defined that can traverse that link with a certain speed and capacity: the link characteristics $C_{a}$. Origins $r \in R$ and destinations $s \in S$ are a subset of $N$ and act as origins 


\section{Table 1. Notation}

\begin{tabular}{|c|c|}
\hline & Transportation network and demand \\
\hline$G(N, A, L, U)$ & Multimodal transportation network, with node set $N$, link set $A$, line set $L$, stop set $U$ \\
\hline$R, S \subseteq N$ & Origin set, destination set \\
\hline$C_{a}$ & Characteristics of link $a$ \\
\hline$A_{l}$ & Subset of links that is traversed by line $l$ \\
\hline$C_{l}$ & Characteristics of line $l$ \\
\hline$C_{u}$ & Characteristics of stop $u$ \\
\hline$M$ & Set of mode chains \\
\hline$K_{r s}, \hat{K}_{m}^{r s}$ & Set of car routes for OD pair $r, s$, set of PT routes for OD pair $r, s$ on mode chain $m$ \\
\hline$f_{k}^{r s}, f_{k m}^{r s}$ & Route flow for car, route flow for PT mode chains \\
\hline$x_{a}, \underline{x}$ & Vehicle link flow, vehicle flow vector \\
\hline$\hat{x}_{a l}, \overline{\hat{x}}$ & Passenger link flow per transit line, passenger flow vector \\
\hline$\delta_{0 k}^{r s}$ & Car route, vehicle link flow indicator \\
\hline$\hat{\delta}_{a l, k m}^{r s}$ & PT route, passenger link and line indicator \\
\hline $\bar{\delta}_{a, k m}^{r s m}$ & PT route, vehicle link indicator \\
\hline$t_{a}\left(x_{a}, \underline{y}\right)$ & Cost function for car links \\
\hline$\hat{t}_{a l}(y)$ & Cost function for PT links per transit line \\
\hline$c_{k}^{r s}, \bar{c}_{k m}^{r s}, \hat{c}_{m}^{r s}$ & Route costs for car, route costs for PT, combined costs for PT mode chains \\
\hline & Combined costs for PT \\
\hline$\zeta_{\mathrm{km}}^{r s}$ & Fraction of travelers on OD pair $r, s$ in PT mode chain $m$, choosing route $k$ \\
\hline$\psi_{m}^{r s}$ & Fraction of PT travelers on OD pair $r, s$ choosing mode chain $m$ \\
\hline $\bar{q}_{r s}, q_{r s}, \hat{q}_{r s}, \hat{q}$ & Total OD demand on, car OD demand, PT OD demand (from $r$ to $s$ ), PT OD demand vector \\
\hline$\theta_{1}, \theta_{2}, \theta_{3}$ & Logit parameters for mode choice, PT mode chain choice, PT route choice \\
\hline & Optimization problem and results \\
\hline$\underline{y}, \underline{y}_{i}^{j}$ & Decision vector, $i^{\text {th }}$ decision vector in pareto set $j$ \\
\hline & Set of feasible decision vectors \\
\hline$V$ & Length of decision vector $y$ \\
\hline$\underline{Z}$ & Objective vector upper level \\
\hline $\bar{z}$ & Objective function lower level \\
\hline$W$ & Length of objective vector $Z$ \\
\hline$J$ & Number of optimization processes \\
\hline$\phi^{j}$ & Set of solutions calculated during optimization process $j$ \\
\hline$P^{j}$ & Pareto set from optimization process $j$ : all non-dominated solutions in $\phi^{j}$ \\
\hline$N^{j}$ & Number of solutions in Pareto set $j$ \\
\hline$\phi^{\Sigma}$ & Set of solutions calculated during all $J$ optimization processes \\
\hline$P^{\Sigma}$ & Superset: : all non-dominated solutions in $\phi^{\Sigma}$ \\
\hline & Genetic parameters \\
\hline$v_{a}$ & Archive size \\
\hline$v_{p}$ & Population size \\
\hline$H$ & Number of generations (index $h$ ) \\
\hline$\pi_{h}$ & Set of archive solutions in generation $h$ \\
\hline$\phi_{h}$ & Set of offspring solutions in generation $h$ \\
\hline$\vartheta_{h}$ & Union of archive and offspring in generation $h$ \\
\hline$\sigma_{h}$ & Set of solutions in mating pool in generation $h$ \\
\hline$\varphi$ & Mutation rate \\
\hline
\end{tabular}

Table 2. Abbreviations

\begin{tabular}{|l|l|}
\hline NSGA-II & Non-dominated Sorting Genetic Algorithm (second version) \\
MOOP & Multi-Objective Optimization Problem \\
NDP & Network Design Problem \\
PT & Public Transport \\
P\&R & Park and Ride \\
IC & Intercity (train) \\
\hline
\end{tabular}


and destinations. Total fixed transportation demand $\bar{q}_{r s}$ is stored in a matrix with size $|\mathrm{R}| \times|\mathrm{S}|$. Furthermore, transit service lines $l \in L$ are defined as ordered subsets $A_{l}$ within $A$ and can be stop services or express services. PT flows can only traverse transit service lines. Transit stations or stops $u \in U$ are defined as a subset within $N$. Consequently, a line $l$ traverses several stops. The travel time between two stops and the frequency of a transit service line $l$ are stored as line characteristics $C_{l}$. Access / egress modes and PT are only connected through these stops. A combination of using a specific access mode, PT and a specific egress mode is defined as a mode chain $M$. Whether a line calls at a stop $u$ or not, is indicated by stop characteristics $C_{u}$. All together, the transportation network is defined by $G(N, A, L, U)$, where $A, L$ and $U$ are further specified by $C_{a}, C_{l}$ and $C_{u}$.

\subsection{Optimization Problem}

We define a decision vector $\underline{y}$ (or a solution), that consists of $V$ decision variables: $\underline{y}=\left\{y_{1}, \cdots, y_{v}, \cdots, y_{v}\right\}, Y$ is the set of feasible values for the decision vector $\underline{y}$ (also called decision space). The objective vector $\underline{Z}$ (consisting of $W$ objective functions, $\left.\underline{Z}=\left\{Z_{1}, \cdots, Z_{w}, \cdots, Z_{w}\right\}\right)$ depends on the value of the decision vector $\underline{y}$. Every $\underline{Z}$ is part of the so called objective space, and in principle $\underline{Z}$ may be any value in $\square^{w}$, but depending on its meaning, an objective function may be subject to natural bounds.

The resulting multi-objective optimization problem (MOOP) is defined in program 1-15. The lower level program in 3-15 is based on the formulation in [21]. The characteristics $C_{a}$, $C_{\text {, }}$ and $C_{u}$ of $A, L$ and $U$ depend on the decision vector $\underline{y}$. These characteristics define the multimodal transportation network $G$. A fixed demand $\bar{q}_{r s}$ is assigned to this transportation network, such that satisfies the constraints (2-3). Feasibility constraint (2) should be satisfied to define a transportation network that is physically within reach. Constraint (3) represents the lower level optimization problem, that optimizes modal split and flows in the network from the traveler's perspective and has equations (4-15) as constraints.

Constraint (4) ensures that the sum of car route flows and transit demand equal total demand. Constraint (5) is a non-negativity constraint for car route flows. Note that PT route flows are nonnegative by the definitions in constraints (6-7). (6) ensures positive PT demand, but bounded by the total demand. (7) distributes PT demand over various mode chains and PT routes, using the fractions in constraints (12-13): (13) defines the route fractions for PT trips per mode chain: the PT model contains multiple routing, where trips are distributed among sensible routes using a logit model. Constraint (12) defines the mode chain fractions for PT trips. Constraint (10) calculates the OD based generalized costs per mode chain as a weighted average of route costs, to correctly take into account combined waiting costs of multiple transit lines. Constraint (9) calculates the logsum for the mode chains, to represent OD based PT costs. Constraints (8) and (11) define the relation between link costs and route costs, for car and for PT. Finally, constraints (14-15) define the vehicle and passenger link flows based on car and PT route flows. Note that PT routes may contain car links, in the case of the use of a P\&R facility in the route (see constraint 14). On the other hand, car routes never include PT passenger links, as can be observed in constraint (8). 


$$
\begin{aligned}
& \min _{\underline{y}} \underline{Z}(\underline{y}, \underline{x}, \underline{\hat{x}}) \quad \text {, s.t. } \\
& \underline{y} \in Y
\end{aligned}
$$

$\underline{x}, \underline{\hat{x}}, \underline{\hat{q}}=\arg \min z(\underline{x}, \underline{\hat{x}}, \underline{\hat{q}})=\sum_{a \in A} \int_{0}^{x_{a}} t_{a}(\omega, \underline{y}) d \omega+\sum_{r \in R, s \in S} \int_{0}^{\hat{q}_{r g}}\left(\frac{1}{\theta_{1}} \ln \frac{\omega}{\bar{q}_{r s}-\omega}+\hat{u}_{r s}\right) d \omega$

$$
\text { s.t. }
$$

$$
\begin{aligned}
& \sum_{k \in K_{r s}} f_{k}^{r s}+\hat{q}_{r s}=\bar{q}_{r s} \\
& f_{k}^{r s} \geq 0
\end{aligned}
$$$$
\forall r, s
$$$$
\forall r, s, k \in K_{r s}
$$$$
0<\hat{q}_{r s}<\bar{q}_{r s}
$$$$
\forall r, s
$$$$
\hat{f}_{k m}^{r s}=\psi_{m}^{r s} \zeta_{k m}^{r s} \hat{q}_{r s}
$$$$
\forall r, s, m, k \in \hat{K}_{m}^{r s}
$$$$
c_{k}^{r s}=\sum_{a \in A} t_{a}\left(x_{a}, \underline{y}\right) \delta_{a, k}^{r s}
$$$$
\forall r, s, k \in K_{r s}
$$$$
\hat{u}_{r s}=-\frac{1}{\theta_{2}} \ln \sum_{m \in M} e^{-\theta_{2} \hat{c}_{m}^{r s}}
$$$$
\forall r, s
$$$$
\hat{c}_{m}^{r s}=\sum_{k \in \hat{K}_{m}^{r s}} \zeta_{k m}^{r s} \hat{c}_{k m}^{r s}
$$$$
\forall r, s, m
$$$$
\hat{c}_{k m}^{r s}=\sum_{a \in A}\left(t_{a}\left(x_{a}, \underline{y}\right) \bar{\delta}_{a, k m}^{r s}+\sum_{l \in L} \hat{t}_{a l}(\underline{y}) \hat{\delta}_{a l, k m}^{r s}\right)
$$$$
\forall r, s, m, k \in \hat{K}_{m}^{r s}
$$$$
\psi_{m}^{r s}=\frac{e^{-\theta_{2} \hat{c}_{m}^{r s}}}{\sum_{m \in M} e^{-\theta_{2} \hat{c}_{m}^{r s}}}
$$$$
\forall r, s, m
$$$$
\forall r, s, m, k \in \hat{K}_{m}^{r s}
$$

$$
\begin{aligned}
& \zeta_{k m}^{r s}=\frac{e^{-\theta_{3} \hat{c}_{b m}^{s s}}}{\sum_{k \in \hat{K}_{m}^{r s}} e^{-\theta_{3} \hat{c}_{b m}^{s s}}} \\
& x_{a}=\sum_{r \in R} \sum_{s \in S} \sum_{k \in\left\{\hat{K}_{n}\right\}} f_{k}^{r s} \delta_{a, k}^{r s}+\sum_{r \in R} \sum_{s \in S} \sum_{m \in M} \sum_{k \in\left\{\hat{K}_{m}^{r m}\right\}} \hat{f}_{l m}^{r s} \bar{\delta}_{a, k m}^{r s} \forall a \\
& \hat{x}_{a l}=\sum_{r \in R} \sum_{s \in S} \sum_{m \in M} \sum_{k \in\left\{\hat{K}_{m}^{n}\right\}} \hat{f}_{k m}^{r s} \hat{\delta}_{a l, l a n}^{r s} \quad \forall a, l
\end{aligned}
$$

Based on the resulting vehicle and passenger flows in the network $G$, an objective vector is calculated for a decision vector $y$ in a general formulation (1). The case specific formulation of objective functions is given separately in Section 4.1.

\subsection{Multi-objective Optimization: Pareto Optimality}

Mathematically, the concept of Pareto optimality is as follows. If we assume two decision vectors $\underline{y}_{i}, \underline{y}_{i}, \in Y$, then $\underline{y}_{i}$ is said to strongly dominate $\underline{y}_{i}$ iff $Z_{w}\left(\underline{y}_{i}\right)<Z_{w}\left(\underline{y}_{i}\right) \forall w$ (also written as $\left.\underline{y}_{i} \prec \underline{y}_{i}\right)$ Additionally, $\underline{y}_{i}$ is said to cover or weakly dominate $\underline{y}_{i}$ iff $Z_{w}\left(\underline{y}_{i}\right) \leq Z_{w}\left(\underline{y}_{i}\right) \forall w$ (also written as $\underline{y}_{i} \underline{y}_{\underline{y}_{i}}$ ). All solutions that are not weakly dominated by another known solution are possibly optimal for the decision maker: these solutions form the Pareto-optimal set $P$. 


\subsection{Solution Method}

The MOOP defined in (1-15) is an NP-hard problem, since the bi-level linear programming problem is already an NP-hard problem [22]. Therefore, the problem is computationally too expensive to be solved exactly for larger networks, so we rely on a heuristic. For solving multi-objective problems, the class of genetic algorithms is often used, because these algorithms have a low risk of ending up in a local minimum, do not require the calculation of a gradient and are able to produce a diverse Pareto set [23].

More specifically, we use the NSGA-II algorithm, developed in [24]. NSGA-II has been successfully applied by researchers to solve multi-objective optimization problems in traffic engineering and proved to be efficient for this type of problems $[2,8]$. It optimizes multiple objectives simultaneously, searching for a set of non-dominated solutions, i.e. the Pareto optimal set. It is a genetic algorithm, based on the principles of natural selection within evolution, combining solutions to new solutions (crossover), where the solutions with a higher fitness value have a larger chance to survive over worse solutions. In the next generation, these enhanced solutions are recombined again, until no progress is made any more or until the maximum number of generations is reached. Within NSGA-II, the mating selection is done by binary tournament selection with replacement. In addition to this mating process, a random mutation operator is applied to a (small) fraction $\varphi$ of solutions from each generation, to promote the exploration of unexplored regions in the decision space. These aspects of the algorithm make that the result is an approximation of the true Pareto set. The random processes in the algorithm cause that from sub sequential runs of the same algorithm, different Pareto sets result. Furthermore, NSGA-II contains elitism, to preserve good solutions in an archive. If the number of non-dominated solutions grows bigger than the archive, the archive only contains the best non-dominated solutions based on the defined fitness value.

The fitness value is calculated in two steps. In the first step (non-dominated sorting), the solutions are ranked based on Pareto dominance. All solutions in the Pareto set receive rank 1. In the next step, these solutions are extracted from the set and all Pareto solutions in the remaining set receive rank 2, etc. In the second step, the solutions are sorted within these ranks based on their crowding distance. Crowding distance calculation requires sorting of the population according to each objective value. The extreme values for each objective are assigned an infinite value, assuring that these values survive. All intermediate solutions are assigned a value equal to the difference in the normalized function values of two adjacent solutions. Concluding, the crowding distance value (and thus the fitness value) is higher if a solutions is more isolated, promoting a more diverse Pareto optimal set. The algorithm in steps (for more details, the reader is referred to [24]):

Step 1: Initialization: Set population size $v_{p}$, which is equal to the archive size $v_{a}$, the maximum number of generations $H$, and generate an initial population $\phi_{0}$. Set. $h=0$ and $\pi_{0}=\varnothing$.

Step 2: Fitness assignment: Combine archive $\pi_{h}$ and offspring $\phi_{h}$, forming $\vartheta_{h}=\pi_{h} \cup \phi_{h}$ and calculate fitness values of solutions by dominance ranking and crowded distance sorting.

Step 3: Environmental selection: Determine new archive $\pi_{h+1}$ by selecting the $v_{a}$ best solutions out of $\vartheta_{h}$ based on their fitness. 
Step 4: Termination: If $h \geq H$ or another stopping criteria is satisfied, determine Pareto set $P$ from the set of all calculated solutions $\phi=\left\{\phi_{0} \cup \cdots \cup \phi_{H}\right\}$ (non-dominated solutions).

Step 5: Mating selection: Perform binary tournament selection with replacement on $\pi_{h+1}$ to determine mating pool of parents $\sigma_{h+1}$.

Step 6: Variation: Apply recombination and mutation operators to the mating pool $\sigma_{g+1}$ to create offspring $\phi_{h+1}$. Set $h=h+1$ and go to step 2 .

\subsection{Output Definitions}

The set $\phi^{j}$ is defined as all decision vectors (or solutions) that are calculated during one optimization process $j$, so $\left|\phi^{j}\right|=v_{a}^{j} H^{j}$. The set of $N^{j}$ solutions $P^{j}=\left\{\underline{y}_{-1}^{j}, \underline{y}_{2}^{j}, \cdots, \underline{y}_{N^{j}}^{j}\right\}$ is defined as the Pareto set resulting from process $j$, which includes all non-dominated solutions with respect to all solutions in $\phi^{j}$, or mathematically there is no $\underline{y}_{i}^{j} \in P^{j}: \underline{y}_{i}^{j} \underline{y}_{y_{i}}^{j}, \forall \underline{y}_{i^{\prime}}^{j} \in \phi^{j} . P^{j}$ is the $j$-th outcome of our MOOP (1): one approximation of the Pareto-optimal set. The $j$-th execution of the algorithm is also denoted as run $j$. Pareto set $P^{\Sigma}$ of all calculated solutions $\phi^{\Sigma}=\left\{\phi^{1} \cup \cdots \cup \phi^{j}\right\}$ is denoted as the superset, and is the best known Pareto set based on $J$ runs.

Please recall that every element or solution $\underline{y}_{i}^{j}$ represents one transportation network design that is defined by the values of $V$ decision variables in $\underline{y}_{i}^{j}$ : $\underline{y}_{i}^{j}=\left\{y_{i 1}^{j}, y_{i 2}^{j}, \cdots, y_{i v}^{j}, \cdots, y_{i V}^{j}\right\}$. For every element $\underline{y}_{i}^{j}$ a vector of objective functions $\underline{Z}\left(\underline{y}_{i}^{j}\right)$ is calculated, that consists of $W$ objective functions, denoted by index $w: \underline{Z}\left(\underline{y}_{i}^{j}\right)=\left\{Z_{1}\left(\underline{y}_{i}^{j}\right), \cdots Z_{w}\left(\underline{y}_{i}^{j}\right), \cdots, Z_{W}\left(\underline{y}_{i}^{j}\right)\right\}$.

\section{Comparing Multiple Optimization Outcomes}

When comparing Pareto sets, it is common to assess the quality of the Pareto sets by indicators. These indicators are usually based on the objective values of the solutions in the Pareto set, to measure either the extent to which the set is close to the real Pareto-optimal front or the extent to which the set is diverse [23].

However, another aspect that is relevant when comparing different outcomes of a randomized algorithm like NSGA-II (and many other MO optimization algorithms), is the extent to which two Pareto sets are similar in the decision space. If a Pareto set is used as a decision support tool, the level of uncertainty in the results is important for the decision maker. Especially the uncertainty in the decision space is of importance, because that is the range he or she can choose from, i.e., this determines if he or she has proper information to make the right decision. The parameter values in the algorithm (in the case of NSGA-II $v_{a}, H$ and $\varphi$ ) may be another source of uncertainty, which is indicated by the sensitivity of the results for these parameters. To be able to assess this similarity formally for our case study in Section 4, in the next section comparison indicators are defined. 


\subsection{Methods for Comparison}

A distinction may be made based on two main characteristics of the method. The first is a distinction between the number of Pareto sets to be compared: a unary indicator can be calculated for one Pareto set on its own, a binary indicator can be calculated for two Pareto sets [25]. The so called attainment function can be calculated for $J$ Pareto sets. The second distinction is whether the method compares the Pareto sets in the objective space or in the decision space: the first type of method is calculated based on the objective function values $\underline{Z}$ of the solutions in the set(s), while the second type of method is calculated based on the decision values $\underline{y}$ of the solutions in the set(s). For comparison in the objective space, a selection of indicators from the literature is presented. For comparison in the decision space, new indicators are defined. An overview of the indicators used in this paper is given in Table 3.

These indicators use a distance function, which can be any distance function defined for two solutions as $d\left(\underline{y}_{i}^{j}, \underline{y}^{j}{ }^{j}\right)$. In this paper the distance function is defined as in formula 16.

$$
d\left(\underline{y}_{i}^{j}, \underline{y}_{i}^{j^{\prime}}\right)=\sum_{v=1}^{V}\left|y_{i v}^{j}-y_{i^{\prime} v}^{j^{\prime}}\right| .
$$

\section{Case Study}

We apply the multi-objective optimization framework to a real life multimodal transportation network, situated in the area around the Dutch cities of Amsterdam and Haarlem (see Figure 2). First, the objectives and decision variables for this specific case are described. After that, the different runs of the NSGA-II algorithm are listed, including their scores on unary performance indicators. Finally, the runs are compared with each other by using the binary indicators and the attainment function.

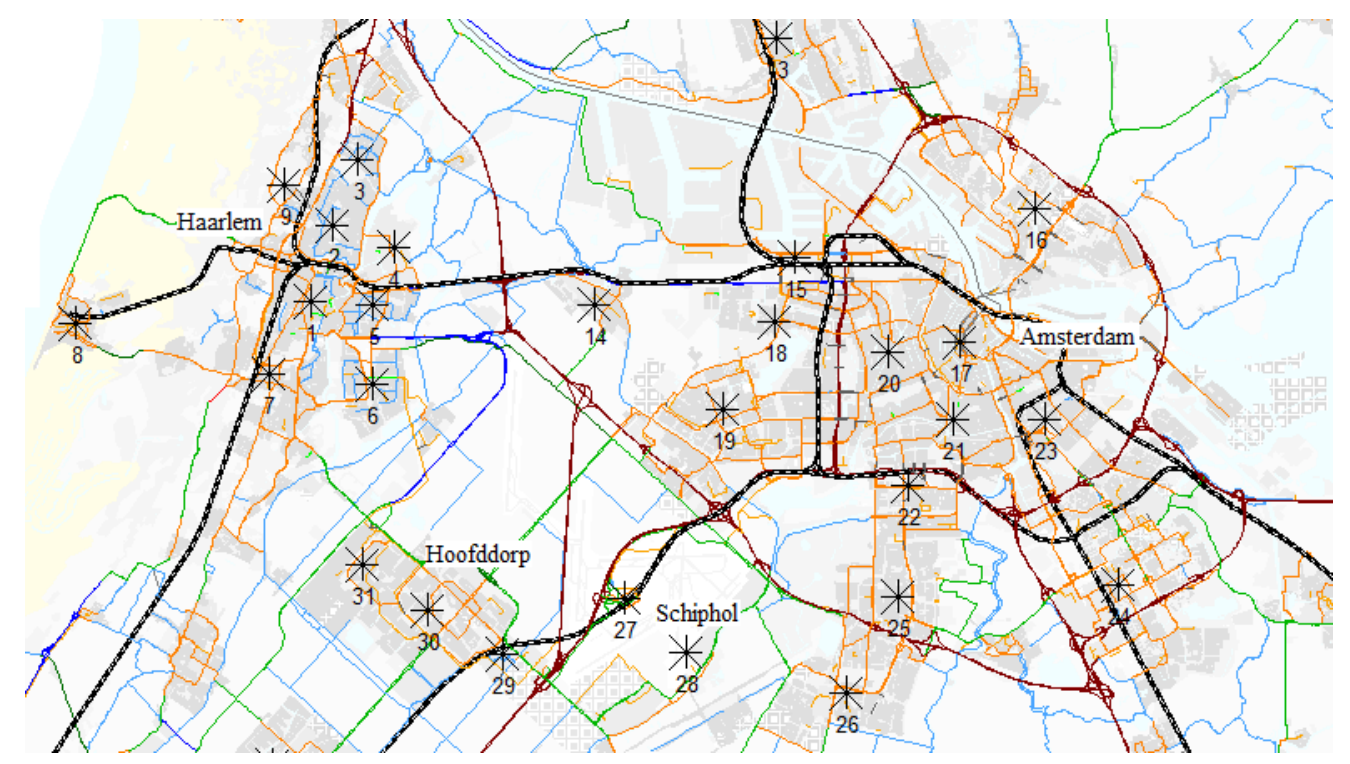

Figure 2. Map of the Study Area, Containing Transportation Zones, Railways and Roads 
Table 3. Overview of Indicators to Compare Pareto Sets

\begin{tabular}{|c|c|c|}
\hline Formula & Type & Description \\
\hline$N^{j}$ & Unary & $\begin{array}{l}\text { Cardinality of Pareto set } j \text {. The bigger a Pareto set, the more } \\
\text { complex it is to analyze for a decision maker. Furthermore, } \\
\text { with more solutions in the set it is easier to fulfill other } \\
\text { criteria, like hypervolume. }\end{array}$ \\
\hline $\begin{array}{l}\operatorname{MIN}_{w}\left(P^{j}\right)=\min _{\underline{y}^{j} \in P^{j}} Z_{w}\left(\underline{y}^{j}\right), \forall w \\
\operatorname{MAX}_{w}\left(P^{j}\right)=\max _{\underline{y}^{j} \in P^{j}} Z_{w}\left(\underline{y}^{j}\right), \forall w \\
R M I_{w}\left(P^{j}\right)=\frac{M I N_{w}\left(P^{j}\right)-M I N_{w}\left(P^{\Sigma}\right)}{M A X_{w}\left(P^{\Sigma}\right)-M I N_{w}\left(P^{\Sigma}\right)}\end{array}$ & $\begin{array}{l}\text { Unary, } \\
\text { objective } \\
\text { space }\end{array}$ & $\begin{array}{l}\text { The minimum objective value attained by Pareto set } j \text { for } \\
\text { every objective function } Z_{w} \text {, with respect to the range } \\
\text { covered by the superset. For that, the minimum and } \\
\text { maximum values are determined for the superset, as well } \\
\text { as the minimum value for set } j \text {. This indicates the extent } \\
\text { to which set } j \text { covers the entire Pareto front for every } \\
\text { objective dimension. }\end{array}$ \\
\hline $\begin{array}{l}\operatorname{SSC}\left(P^{k}\right) \\
\text { Fig. 3: hypervolume 2-D visualization }\end{array}$ & $\begin{array}{l}\text { Unary, } \\
\text { objective } \\
\text { space }\end{array}$ & $\begin{array}{l}\text { The space coverage. Implemented as in [26], also known } \\
\text { as S-metric or hypervolume. In the 2-dimensional case it } \\
\text { determines the area that is covered by the Pareto set with } \\
\text { respect to a reference point (the star in fig. 3). The } \\
\text { reference point represents the upper bound of all } \\
\text { objectives: the reference point is defined such that it is } \\
\text { dominated by all solutions in the Pareto set. Because the } \\
\text { true maximum values of the objective functions are not } \\
\text { known, we choose a conservative point, based on the } \\
\text { evaluated solutions. In the } 3 \text { dimensional case area is } \\
\text { replaced by volume, and in the more dimensional case } \\
\text { by hypervolume. }\end{array}$ \\
\hline $\operatorname{CTS}\left(P^{j}, P^{j^{\prime}}\right)=\frac{\left\{\underline{y}^{j^{\prime}} \in P^{j^{\prime}} ; \exists \underline{y}^{j} \in P^{j}: \underline{y}^{j} \preceq \underline{y}^{j^{\prime}}\right\} \mid}{N^{j^{\prime}}}$ & $\begin{array}{l}\text { Binary, } \\
\text { objective } \\
\text { space }\end{array}$ & $\begin{array}{l}\text { The set coverage or C-metric, see [27]. The level in } \\
\text { which the solutions in } P^{j^{\prime}} \text { are weakly dominated by at } \\
\text { least one solution in set } P^{j} \text {, so a higher value indicates } \\
\text { a better set coverage of } P^{j} \text { over } P^{j^{\prime}} \text {. }\end{array}$ \\
\hline$A D\left(P^{j}, P^{j^{\prime}}\right)=\frac{1}{N_{j}} \frac{1}{N_{j^{\prime}}} \sum_{i=1}^{N_{j}} \sum_{i^{\prime}=1}^{N_{j}} d\left(\underline{y}_{i}^{j}, \underline{y}_{i^{\prime}}^{j^{\prime}}\right)$ & $\begin{array}{l}\text { Binary, } \\
\text { decision } \\
\text { space }\end{array}$ & $\begin{array}{l}\text { The average distance (in the decision space) between the } \\
\text { solutions in two Pareto sets } P^{j} \text { and } P^{j^{\prime}} \text { (where } j \text { may } \\
\text { also be equal to } j^{\prime} \text { ). This indicator is used to check } \\
\text { whether the variation within a Pareto set is different } \\
\text { from the variation between Pareto sets. }\end{array}$ \\
\hline$A N D\left(P^{j}, P^{j^{\prime}}\right)=\frac{1}{N^{j}} \sum_{i=1}^{N^{j}} \min _{i^{\prime} \in\left\{1 \ldots N^{\prime}\right\}} d\left(\underline{y}_{-i}^{j}, \underline{y}_{i}^{j^{\prime}}\right)$ & $\begin{array}{l}\text { Binary, } \\
\text { decision } \\
\text { space }\end{array}$ & $\begin{array}{l}\text { The distance of an element in } P^{j} \text { to the closest element (i.e. } \\
\text { most similar in decision space) in Pareto set } P^{j^{\prime}} \text {, averaged } \\
\text { over all elements } P^{j} \text {. A low value indicates higher } \\
\text { similarity (the extreme case } \operatorname{AND}\left(P^{j}, P^{j}\right)=0 \text { holds). }\end{array}$ \\
\hline $\begin{array}{l}\text { AFD }\left(P^{j}, P^{j^{\prime}}\right)=\frac{1}{V} \sum_{i=1}^{V}\left|\rho_{v}^{j}-\rho_{v}^{j^{\prime}}\right| \\
\rho_{v}^{j}=\frac{1}{N^{j}} \sum_{i=1}^{N^{j}} \kappa\left(y_{i v}^{j}\right), \text { with } \\
\kappa\left(y_{i v}^{j}\right)= \begin{cases}0 & \text { if } y_{i v}^{j}=0 \\
1 & \text { otherwise }\end{cases} \end{array}$ & $\begin{array}{l}\text { Binary, } \\
\text { decision } \\
\text { space }\end{array}$ & $\begin{array}{l}\text { Comparison of fractions of nonzero decision variables. The } \\
\text { fraction } \rho_{v}^{j} \text { of solutions in Pareto set } j \text { that have a positive } \\
\text { value for decision variable } y_{v} \text { characterizes the decision } \\
\text { space of the set: decision variable } y_{v} \text { represents the } \\
\text { existence of a measure in the transportation network, so a } \\
\text { higher fraction for variable } y_{v} \text { implies a larger ability to } \\
\text { attain Pareto solutions when that variable has a positive } \\
\text { value. The } \kappa \text { function defines the nonzero relation. To } \\
\text { indicate the difference between Pareto set } j \text { and } j \text {, the }\end{array}$ \\
\hline
\end{tabular}




\begin{tabular}{|l|l|l|}
\hline$\alpha(\underline{Z})=\frac{1}{J} \sum_{j=1}^{J} I\left(\exists \underline{y^{j}} \in P^{j}: \underline{y} \underline{\underline{Z}} \underline{\underline{Z}}\right)$ & $\begin{array}{l}\text { differences between these fractions are calculated, averaged } \\
\text { over all } V \text { decision variables. }\end{array}$ \\
\hline $\begin{array}{l}\text { Compare } \\
J \quad \text { Pareto } \\
\text { sets, } \\
\text { objective } \\
\text { space }\end{array}$ & $\begin{array}{l}\text { vector } \underline{Z} \text { this function returns the probability that } \underline{Z} \text { is } \\
\text { attained by a fraction } \alpha \text { of } J \text { Pareto sets. It is approximated } \\
\text { on basis of the approximation set sample. Function } \\
I \text { returns } 1 \text { if its argument is true and } 0 \text { if it is false. }\end{array}$ \\
\hline
\end{tabular}

\subsection{Objectives}

In this paper we consider 4 policy objectives related to sustainability, concerning accessibility, use of urban space by parking, climate impact and costs. These objectives and their operationalization are shown in table 4. Variables that are not defined earlier, are defined below the table. All 4 objectives are to be minimized. In our case of a fixed total demand, total travel time gives the same results as average travel time would have given. The urban space used by parking is represented by the number of car trips to or from zones that are classified as highly urban, because such a trip requires a parking space that cannot be used for other urban land uses. These alternative land uses give additional value to property [28]. $\mathrm{CO}_{2}$ emissions are calculated using the ARTEMIS traffic situation based emission model, where emission factors for cars on a link depend on free flow speed and volume / capacity ratio [29]. Emission factors for PT depend on vehicle type. Operating deficit of the PT system is formulated as an objective, rather than as a budget constraint, to provide explicit insight in the relation between costs and other objectives. Cost parameters follow from Dutch PT operating practice.

\subsection{Decision Variables}

Decision variables in this case study are related to transfer facilities or to PT facilities. For every potential network development, a decision variable $y_{v}$ is defined in advance (see Table 5). Network developments are only included as a candidate location if spatial and capacity constraints are met. For example, a P\&R location is only opened if the corresponding station is served by PT. The characteristics of links, lines and stops that are not candidate locations are fixed at one value. Furthermore, the car and bicycle networks are assumed to be fixed. In this case, the feasible region $Y$ contains approximately $10^{9}$ possible decision vectors.

\section{Frequency of PT Service Lines}

A part of the decision vector $y$ represents frequencies of PT service line, including train lines (local trains stop at all stations they pass through, Intercity (IC) trains or express trains only stop at IC stations) and main bus lines (with multiple stops). In our case, the frequencies can be chosen from $\{0,2,4,6\}$, so the value may also be 0 : the line does not exist in that case. To achieve comparable values of $y_{v}$ over the entire the decision space, the corresponding values that $y_{v}$ are normalized as $\left\{0, \frac{1}{3}, \frac{2}{3}, 1\right\}$. Tram line extensions are defined as a binary variable, indicating whether the line is extended or not (with the original frequency). A feeder bus network is available in the network, but is assumed to be fixed in this case.

\section{Existence of Train Stations}

For the existence of train stations $y_{v}$ is defined as a binary variable, that indicates whether transit vehicles call at a stop $u$ or not (i.e. exchange between the walk and bicycle networks 
and the PT network). Note that for bus stops and for existing train stations the value of $y_{v}$ is fixed to 1: these PT stops can in all solutions be reached by bicycle and walking.

\section{Express status of train station $u$}

For the express train status of train stations, $y_{v}$ is defined as a binary variable, that indicates whether transit vehicles of express lines call at a stop $u$ or not. Note that at existing express stops this variable is fixed to 1 .

\section{Table 4. Objectives and their Operationalization in Words and in Mathematical Formulation}

\begin{tabular}{|c|c|c|}
\hline $\begin{array}{l}\text { Aspect of } \\
\text { sustainability }\end{array}$ & Operationalized by & Formulation \\
\hline Accessibility & $\begin{array}{l}\text { Total (generalized) } \\
\text { travel time }\end{array}$ & $Z_{1}=\sum_{r \in R, s \in S} c_{r s} q_{r s}+\sum_{r \in R, s \in S} \sum_{m \in M} \hat{c}_{m}^{r s} \psi_{m}^{r s} \hat{q}_{r s}$ \\
\hline $\begin{array}{l}\text { Use of urban } \\
\text { space by parking }\end{array}$ & \begin{tabular}{l|l} 
Ig & $\begin{array}{l}\text { Number car trips to } \\
\text { and from urban } \\
\text { zones }\end{array}$ \\
\end{tabular} & $Z_{2}=\sum_{r \in R_{U}} \sum_{s \in S}\left(q_{r s}+\sum_{m \in M_{R}} \psi_{m}^{r s} \hat{q}_{r s}\right)+\sum_{r \in R} \sum_{s \in S_{U}}\left(q_{r s}+\sum_{m \in M_{S}} \psi_{m}^{r s} \hat{q}_{r s}\right)$ \\
\hline Climate impact & $\mathrm{CO}_{2}$ emissions & $Z_{3}=\sum_{a \in A} x_{a} E^{C O_{2}}\left(v_{a}^{0}, \frac{x_{a}}{x_{a}^{\max }}\right) d_{a}+\sum_{a \in A} \sum_{b \in B} \sum_{l \in L} \Delta_{b l} x_{a l} \hat{E}_{b}^{C O_{2}} d_{a}$ \\
\hline Cost efficiency & $\begin{array}{l}\text { PT operating deficit } \\
\text { (operating costs - } \\
\text { operating revenues) }\end{array}$ & $Z_{4}=\sum_{b \in B} \sum_{l \in L}\left[\Delta_{b l} O_{b} \sum_{a \in A_{j}} x_{a l} t_{a l}\right]-\sum_{b \in B} \sum_{l \in L}\left[\Delta_{b l} f_{b} \sum_{a \in A_{j}} d_{a} \hat{x}_{a l}\right]$ \\
\hline \multicolumn{3}{|l|}{ With: } \\
\hline \begin{tabular}{l|}
$x_{a l} E^{C O_{2}}\left(v_{a}^{0}, \frac{x_{a}}{x_{a}^{\max }}\right)$ \\
$\hat{E}_{b}^{C O_{2}}$ \\
$d_{a}$ \\
$R_{U}, S_{U}$ \\
$M_{R}$ \\
$M_{S}$ \\
$B$ \\
$O_{b}$ \\
$\Delta_{b l}$ \\
$f_{b}$
\end{tabular} & \multicolumn{2}{|c|}{$\begin{array}{l}\text { Vehicle flow on link } a \text { for transit line } l \text { (veh per hour), determined by network definition. } \\
\mathrm{CO}_{2} \text { emission factor for cars, depending on free flow speed } v_{a}^{0} \text { of link } a \text { and volume / } \\
\text { capacity ratio } \frac{x_{a}}{x_{a}^{\max } \text { of link } a \text { (grams/(veh*km)). }} \\
\mathrm{CO}_{2} \text { emission factor for PT vehicle type } b \text {. } \\
\text { Length of link } a(\mathrm{~km}) \\
\text { Set of highly urban origins, set of highly urban destinations } \\
\text { Set of mode chains that start the trip with a car leg } \\
\text { Set of mode chains that end the trip with a car leg } \\
\text { Set of PT vehicle types } \\
\text { Operating costs for PT vehicle type } b \text { (euros per vehicle*hour) } \\
\text { PT vehicle type indicator, equals } 1 \text { if line } l \text { is of vehicle type } b, 0 \text { otherwise } \\
\text { Fare for using PT of vehicle type } b \text { (euros per km) }\end{array}$} \\
\hline
\end{tabular}

Park and Ride facility at stop $u$

For Park and Ride, $y_{v}$ is defined as a binary variable that indicates whether it is possible to reach a stop by car, by changing the characteristics of the link connecting the stop to the access network. Note that there are also existing P\&R facilities that are fixed to 1, i.e., PT stops that can in all solutions be reached by car.

\subsection{Pareto Set Approximations}

The NSGA-II algorithm was executed 13 times in total (see Table 6). The first 5 runs were executed with identical parameter settings, to investigate the impact of randomness in the algorithm. After that, in two more groups of 3 runs, 2 different combinations of parameter settings were chosen. Finally, two unique runs were executed, with a different mutation rate. A set of runs with the same parameter settings is further denoted as a group of runs. The groups are highlighted in Table 6. All parameter settings were chosen in such a way that 
computation times were still reasonable (one week per run), while convergence was reached or almost reached in terms of attained hypervolume. Furthermore, for a fair comparison, the total computation time (indicated by the total number of calculated solutions $v_{a} H$ ) was approximately the same for all runs.

Table 5. The Definition of 26 Decision Variables in the Case Study

\begin{tabular}{|c|c|c|c|c|}
\hline $\begin{array}{l}\text { Decision } \\
\text { variable } \\
\text { index } v\end{array}$ & $\begin{array}{l}\text { Possible } \\
\text { values of } \\
y_{v}\end{array}$ & $\begin{array}{l}\text { Represents } \\
\text { real value }\end{array}$ & Description of decision variable & $\begin{array}{l}\text { Fraction of } \\
\text { nonzero values } \\
\text { in superset } \rho_{v}^{\Sigma}\end{array}$ \\
\hline 1 & $\left\{\frac{1}{3}, \frac{2}{3}\right\}$ & $\{2,4\}$ & $\begin{array}{l}\text { Frequency of intercity train Amsterdam - Haarlem - } \\
\text { Leiden }\end{array}$ & $100 \%$ \\
\hline 2 & $\{0,1\}$ & $\{0,2\}$ & $\begin{array}{l}\text { Frequency of local train Amsterdam - Haarlem - } \\
\text { Leiden }\end{array}$ & $16 \%$ \\
\hline 3 & $\left\{0, \frac{1}{3}, \frac{2}{3}, 1\right\}$ & $\{0,2,4,6\}$ & $\begin{array}{l}\text { Frequency of new train line Uitgeest - Haarlem - } \\
\text { Amsterdam Sloterdijk - Zuid - Bijlmer }\end{array}$ & $75 \%$ \\
\hline 4 & $\{0,1\}$ & $\{0,2\}$ & $\begin{array}{l}\text { Frequency of train line Uitgeest - Haarlem - } \\
\text { Amsterdam Centraal }\end{array}$ & $51 \%$ \\
\hline 5 & $\{0,1\}$ & $\{0,2\}$ & Frequency of train line Haarlem - Leiden & $14 \%$ \\
\hline 6 & $\left\{0, \frac{1}{3}, \frac{2}{3}, 1\right\}$ & $\{0,2,4,6\}$ & $\begin{array}{l}\text { Frequency of bus line 176: Amsterdam Zuid - } \\
\text { Haarlem }\end{array}$ & $76 \%$ \\
\hline 7 & $\left\{0, \frac{1}{3}, \frac{2}{3}, 1\right\}$ & $\{0,2,4,6\}$ & $\begin{array}{l}\text { Frequency of bus line 83: IJmuiden - A'dam } \\
\text { Sloterdijk }\end{array}$ & $99 \%$ \\
\hline 8 & $\left\{0, \frac{1}{3}, \frac{2}{3}, 1\right\}$ & $\{0,2,4,6\}$ & $\begin{array}{l}\text { Frequency of new bus line Hoofddorp - Amsterdam } \\
\text { West - Sloterdijk }\end{array}$ & $53 \%$ \\
\hline 9 & $\left\{0, \frac{1}{3}, \frac{2}{3}, 1\right\}$ & $\{0,2,4,6\}$ & $\begin{array}{l}\text { Frequency of bus line 175: Haarlem - Amstelveen - } \\
\text { Amsterdam Zuidoost }\end{array}$ & $16 \%$ \\
\hline 10 & $\left\{0, \frac{1}{3}, \frac{2}{3}, 1\right\}$ & $\{0,2,4,6\}$ & $\begin{array}{l}\text { Frequency of bus line 80: Zandvoort - Haarlem - } \\
\text { Amsterdam Marnixstraat }\end{array}$ & $74 \%$ \\
\hline 11 & $\left\{0, \frac{1}{3}, \frac{2}{3}, 1\right\}$ & $\{0,2,4,6\}$ & $\begin{array}{l}\text { Frequency of new bus line 'Westtangent': Amsterdam } \\
\text { Sloterdijk - Amsterdam West - Schiphol }\end{array}$ & $62 \%$ \\
\hline 12 & $\{0,1\}$ & Existence & Extention of tram 13 in Geuzenveld & $42 \%$ \\
\hline 13 & $\{0,1\}$ & Existence & Extention of express tram Amstelveen to Uithoorn & $62 \%$ \\
\hline 14 & $\{0,1\}$ & Existence & New train station Halfweg-Zwanenburg & $1 \%$ \\
\hline 15 & $\{0,1\}$ & Existence & New train station Haarlem Zuid & $36 \%$ \\
\hline 16 & $\{0,1\}$ & Existence & New train station Amsterdam Geuzenveld & $0 \%$ \\
\hline 17 & $\{0,1\}$ & no ICs, ICs & Intercity status of station Hoofddorp & $88 \%$ \\
\hline 18 & $\{0,1\}$ & no ICs, ICs & Intercity status of station Heemstede-Aardenhout & $7 \%$ \\
\hline 19 & $\{0,1\}$ & no ICs, ICs & Intercity status of station Amsterdam Lelylaan & $22 \%$ \\
\hline 20 & $\{0,1\}$ & no ICs, ICs & Intercity status of station Duivendrecht & $45 \%$ \\
\hline 21 & $\{0,1\}$ & Existence & $P \& R$ at train station Halfweg-Zwanenburg & $0 \%$ \\
\hline 22 & $\{0,1\}$ & Existence & P\&R Velsen Zuid (at bus line 83) & $30 \%$ \\
\hline 23 & $\{0,1\}$ & Existence & P\&R at tram station Amstelveen Oranjebaan & $2 \%$ \\
\hline 24 & $\{0,1\}$ & Existence & P\&R at train station Geuzenveld and tram 13 & $5 \%$ \\
\hline 25 & $\{0,1\}$ & Existence & $P \& R$ Badhoevedorp at new bus line & $12 \%$ \\
\hline 26 & $\{0,1\}$ & Existence & $\begin{array}{l}\text { P\&R Schiphol Noord at bus lines 'Westtangent' and } \\
\text { 'Zuidtangent' }\end{array}$ & $30 \%$ \\
\hline
\end{tabular}


Table 6. Parameter Settings and Unary Indicators of the 13 Runs of the Optimization Process

\begin{tabular}{|r|r|r|r|r|r|}
\hline $\begin{array}{l}\text { Set } \\
\text { number } j\end{array}$ & $\begin{array}{l}\text { archive } \\
\text { size } v_{a}\end{array}$ & $\begin{array}{l}\text { Number of } \\
\text { generations } H\end{array}$ & $\begin{array}{l}\text { Mutation } \\
\text { rate } \varphi\end{array}$ & $\begin{array}{l}\text { Number of } \\
\text { solutions in set } \\
N^{j}\end{array}$ & $\begin{array}{l}\text { Hypervolume } \\
S S C\left(P^{j}\right) \\
\left(* 10^{21}\right)\end{array}$ \\
\hline 1 & 80 & 36 & 0.03 & 339 & 9.57 \\
2 & 80 & 36 & 0.03 & 282 & 9.78 \\
3 & 80 & 36 & 0.03 & 301 & 9.84 \\
4 & 80 & 36 & 0.03 & 364 & 9.76 \\
5 & 80 & 36 & 0.03 & 291 & 9.82 \\
\hline 6 & 50 & 60 & 0.03 & 287 & 9.80 \\
7 & 50 & 60 & 0.03 & 329 & 9.88 \\
8 & 50 & 60 & 0.03 & 340 & 9.76 \\
\hline 9 & 100 & 30 & 0.03 & 363 & 9.86 \\
10 & 100 & 30 & 0.03 & 255 & 9.79 \\
11 & 100 & 30 & 0.03 & 312 & 9.85 \\
\hline 12 & 80 & 36 & 0.1 & 159 & 9.28 \\
\hline 13 & 80 & 36 & 0.01 & 337 & 9.80 \\
\hline$\Sigma$ & - & - & - & 1019 & 10.12 \\
\hline
\end{tabular}

\subsection{Unary Performance Indicators}

All 13 runs were able to attain considerably larger hypervolumes (Table 6) than the random starting populations (the average value of hypervolume covered by the starting populations $\phi_{0}^{1}$ to $\phi_{0}^{J}$ was $7.31 * 10^{21}$ ). Furthermore, the individual runs are not too far from the best known Paretoset, the superset $P^{\Sigma}$, taking into account that the superset contains a larger number of solutions than the individual runs.

When we compare the number of attained Pareto solutions, only run 12 shows a large difference with all other runs. The variation within the number of Pareto solutions is similar within groups of runs and between groups of runs. The same holds for the hypervolume that is covered by the Pareto sets. In addition, run 1 has a relatively low value for hypervolume, which seems to be an outlier resulting from the random optimization process.

Next, the minimum values attained by the different runs $R M I_{w}\left(P^{j}\right)$ for all 4 objective functions are compared. Some observations from Figure 4 are:

- $\quad$ Travel time has the most (relative) variation from all objectives

- $\quad$ Run 12 does not provide (near) optimal solutions for any objective

- $\quad$ Run 7 performs excellent, achieving 3 minimal values and 1 average value

Although no hard conclusions can be drawn based on one optimization outcome, the unary indicators show that $P^{12}$ has a worse performance than the other Pareto sets. This is also shown by the set coverage with respect to $P^{12}$ : the average value over $j, j \neq 12$ of $\operatorname{CTS}\left(P^{j}, P^{12}\right)$ is 0.94 , while the average value over $j, j \neq 12$ of $\operatorname{CTS}\left(P^{12}, P^{j}\right)$ is 0.43 . This indicated that a mutation rate of 0.1 (as was applied in run 12) is too high for this type of problem. 


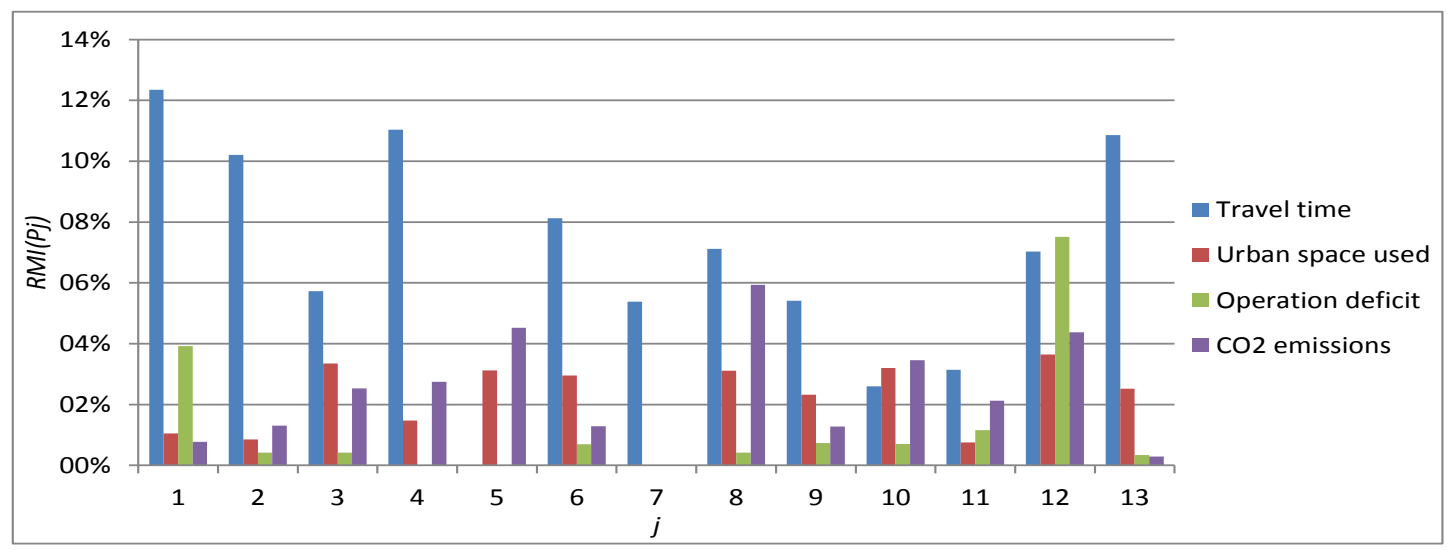

Figure 4. The Relative Values $R M I_{w}\left(P^{j}\right)$, for 13 Runs and for 4 Objectives

\subsection{Comparing Pairs of Pareto Sets}

In this section a pairwise comparison between Pareto sets is made. Firstly, Pareto sets 2 and 3 are plotted as an example in fig. 5. This shows that the sets have comparable shapes, but the extremes are different, and one Pareto set contains more solutions in certain areas than the other set. Next, the indicators defined in Section 3.1 are used to compare the Pareto sets.

Table 7 shows the values of $A D\left(P^{j}, P^{j^{\prime}}\right)$ for each pair of Pareto sets. The diagonal of the table contains the values for the average distance within the same Pareto set. No clear differences can be observed in the table. However, there is a small difference between cases where $j=j^{\prime}$ and cases where $j \neq j^{\prime}$ : overall, these average values are 6.5 resp. 6.8 . Furthermore, there is a small difference between the average value within groups of runs (6.7) and between groups of runs (6.9). These differences seem too small to be substantial, although one would expect that a pair of Pareto sets with the same parameter settings would have more similarities than a pair of Pareto sets with different parameter settings.
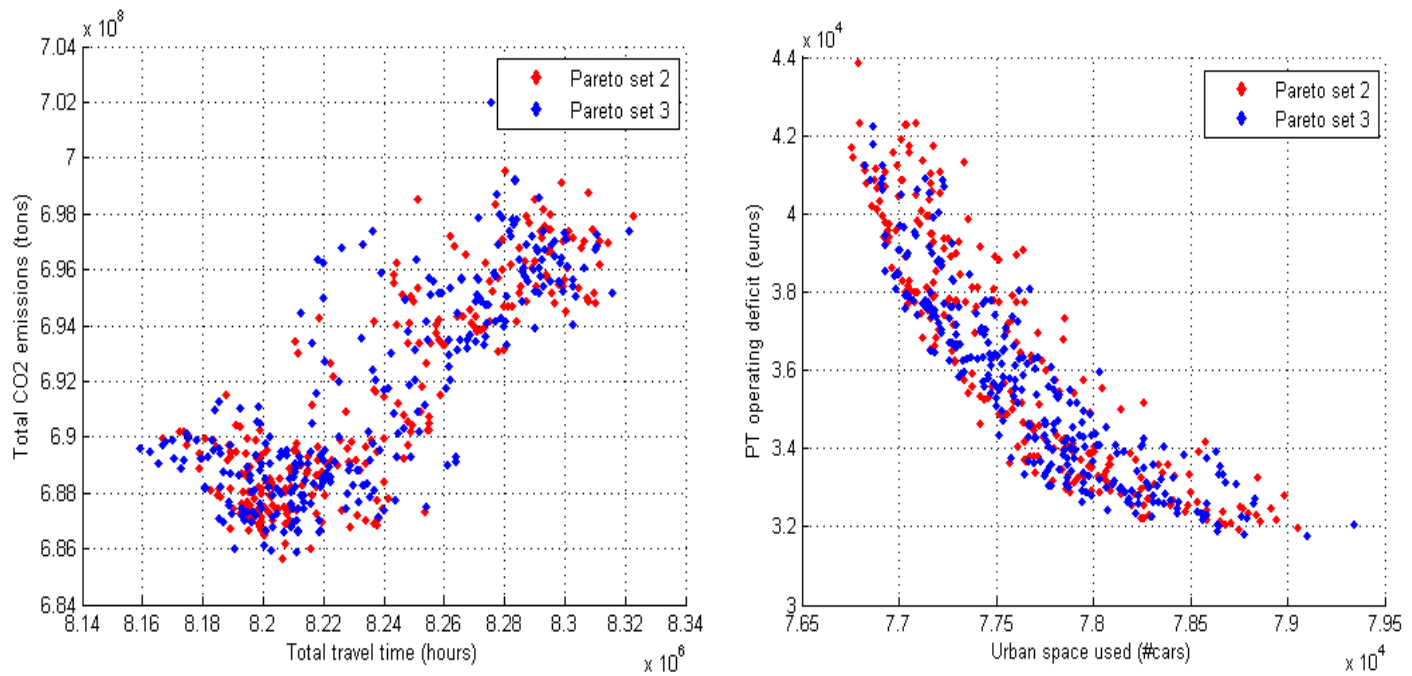

Figure 5. 2 Dimensional Plots of Two Different Outcomes of the Optimization Process, for Objective 1 and 3 (Left) and for Objective 2 and 4 (Right) 
Table 7. The Average Distance between Pareto Solutions ${ }^{A D\left(P^{j}, P^{j^{\prime}}\right)}$

\begin{tabular}{|c|c|c|c|c|c|c|c|c|c|c|c|c|c|}
\hline & 1 & 2 & 3 & 4 & 5 & 6 & 7 & 8 & 9 & 10 & 11 & 12 & 13 \\
\hline 1 & 6.4 & 6.9 & 6.9 & 6.9 & 7.1 & 6.7 & 7.2 & 6.9 & 7.1 & 7.0 & 7.0 & 7.6 & 7.0 \\
\hline 2 & & 6.5 & 6.5 & 6.6 & 6.7 & 6.6 & 7.0 & 6.8 & 6.7 & 6.8 & 6.7 & 7.5 & 6.8 \\
\hline 3 & & & 5.8 & 6.4 & 6.3 & 6.4 & 6.6 & 6.6 & 6.4 & 6.2 & 6.4 & 7.3 & 6.4 \\
\hline 4 & & & & 6.4 & 6.7 & 6.7 & 6.9 & 6.9 & 6.8 & 6.7 & 6.7 & 7.6 & 6.8 \\
\hline 5 & & & & & 6.4 & 6.6 & 6.8 & 6.7 & 6.5 & 6.7 & 6.6 & 7.6 & 6.8 \\
\hline 6 & & & & & & 6.1 & 6.7 & 6.5 & 6.6 & 6.6 & 6.7 & 7.4 & 6.6 \\
\hline 7 & & & & & & & 6.6 & 6.9 & 6.8 & 6.9 & 6.8 & 7.7 & 7.0 \\
\hline 8 & & & & & & & & 6.4 & 6.6 & 6.8 & 6.7 & 7.5 & 6.8 \\
\hline 9 & & & & & & & & & 6.2 & 6.7 & 6.5 & 7.4 & 6.8 \\
\hline 10 & & & & & & & & & & 6.5 & 6.7 & 7.4 & 6.7 \\
\hline 11 & & & & & & & & & & & 6.5 & 7.6 & 6.8 \\
\hline 12 & & & & & & & & & & & & 7.8 & 7.5 \\
\hline 13 & & & & & & & & & & & & & 6.6 \\
\hline
\end{tabular}

Table 8 shows the values of $A N D\left(P^{j}, P^{j^{\prime}}\right)$ for each pair of Pareto sets. Note that the diagonal only contains zeros: in the same Pareto set an identical solution with $d\left(\underline{y}_{i}^{j}, \underline{y}_{i}^{j^{\prime}}\right)=0$ can always be found. Another observation is that the indicator is not symmetrical, i.e., $A N D\left(P^{j}, P^{j^{\prime}}\right) \neq A N D\left(P^{j^{\prime}}, P^{j}\right):$ a solution $\underline{y}_{i}^{j}$ in set $j$ that acts as nearest solution for solution $\underline{y}_{i^{\prime}}{ }^{\prime \prime}$ may not have solution $\underline{y}_{-}^{j^{\prime}}{ }^{\prime}$ as nearest solution in set $j^{\prime}$. Again, only minor differences can be observed in the table. However, the absolute values of $A N D\left(P^{j}, P^{j^{\prime}}\right)$ have an implication: on average, the closest solution in another Pareto optimal set can be found at a distance of 2.3. This implies that on average more than 2 of 26 the decision variables are different if a solution from another random outcome of the optimization process is used, which is a substantial difference. Furthermore, also in this indicator there is a small difference between the average value within groups of runs (2.2) and between groups of runs (2.3). These differences seem too small to substantial.

Table 8. The Average Distance to the Nearest Pareto Solution $\operatorname{AND}\left(P^{j}, P^{j^{\prime}}\right)$

\begin{tabular}{|r|rrrrr|rrr|rrr|r|r|}
\cline { 2 - 12 } \multicolumn{1}{c|}{} & 1 & 2 & 3 & 4 & 5 & 6 & 7 & 8 & 9 & 10 & 11 & 12 & 13 \\
\hline 1 & 0.0 & 2.3 & 2.7 & 2.2 & 2.6 & 2.4 & 2.6 & 2.5 & 2.5 & 2.6 & 2.5 & 3.1 & 2.4 \\
2 & 2.3 & 0.0 & 2.3 & 2.1 & 2.2 & 2.2 & 2.4 & 2.2 & 2.2 & 2.3 & 2.1 & 2.9 & 2.1 \\
3 & 2.5 & 2.0 & 0.0 & 1.9 & 1.8 & 2.0 & 2.0 & 2.0 & 2.0 & 1.9 & 1.9 & 2.7 & 1.8 \\
4 & 2.3 & 2.2 & 2.2 & 0.0 & 2.2 & 2.4 & 2.4 & 2.4 & 2.3 & 2.2 & 2.1 & 2.9 & 2.2 \\
5 & 2.4 & 2.0 & 2.1 & 2.0 & 0.0 & 2.2 & 2.0 & 2.0 & 1.9 & 2.3 & 1.8 & 3.0 & 2.1 \\
\hline 6 & 2.3 & 2.1 & 2.1 & 2.2 & 2.2 & 0.0 & 2.1 & 2.0 & 2.1 & 2.2 & 2.1 & 2.7 & 2.0 \\
7 & 2.4 & 2.3 & 2.3 & 2.1 & 2.1 & 2.2 & 0.0 & 2.3 & 2.2 & 2.4 & 2.1 & 2.9 & 2.3 \\
8 & 2.4 & 2.1 & 2.2 & 2.3 & 2.1 & 2.0 & 2.2 & 0.0 & 1.8 & 2.4 & 2.0 & 2.8 & 1.9 \\
\hline 9 & 2.5 & 2.1 & 2.1 & 2.1 & 1.9 & 2.1 & 2.1 & 1.8 & 0.0 & 2.2 & 1.8 & 2.7 & 2.0 \\
10 & 2.5 & 2.3 & 2.1 & 2.1 & 2.2 & 2.3 & 2.4 & 2.2 & 2.1 & 0.0 & 2.2 & 2.7 & 2.0 \\
11 & 2.5 & 2.1 & 2.1 & 2.0 & 2.0 & 2.2 & 2.3 & 2.2 & 1.9 & 2.2 & 0.0 & 2.8 & 2.1 \\
\hline 12 & 3.2 & 3.2 & 3.2 & 3.0 & 3.2 & 3.1 & 3.1 & 3.0 & 2.9 & 3.0 & 3.0 & 0.0 & 3.0 \\
\hline 13 & 2.3 & 2.1 & 2.1 & 2.2 & 2.2 & 2.1 & 2.3 & 2.0 & 2.1 & 2.0 & 2.1 & 2.7 & 0.0 \\
\hline
\end{tabular}


Table 9. The Difference between Fractions of Nonzero Decision Variables in the Pareto Sets $A F D\left(P^{j}, P^{j^{\prime}}\right)$

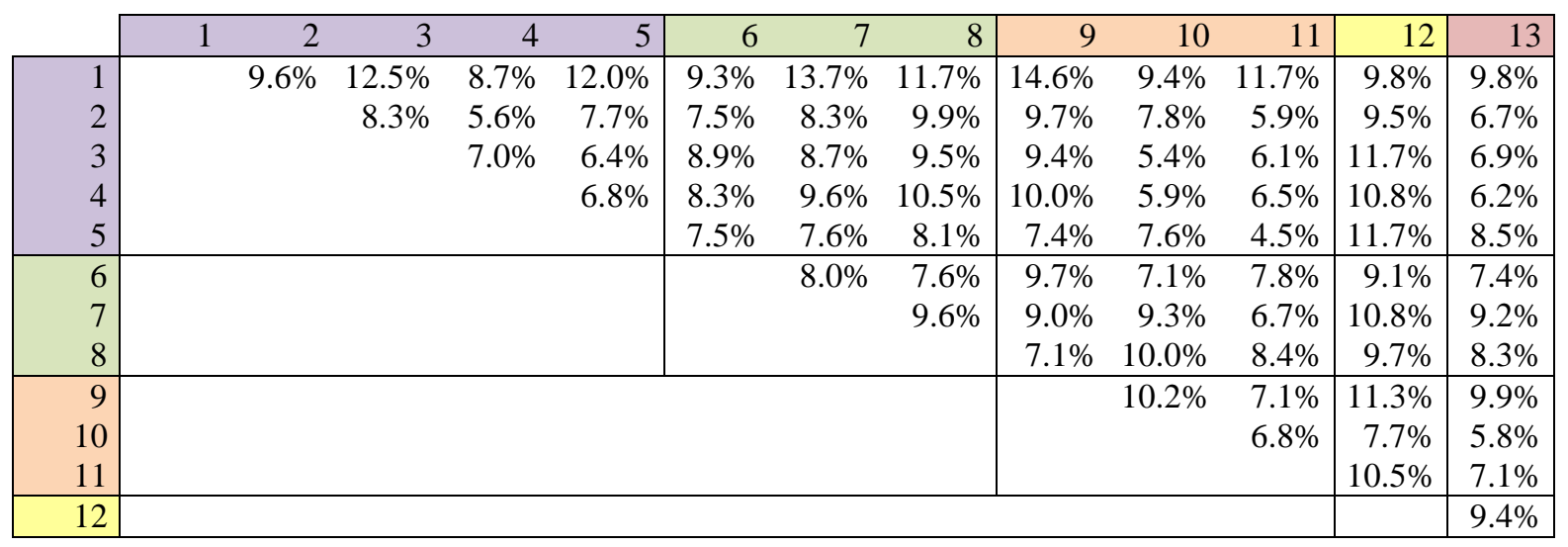

Table 9 contains the values for $A F D\left(P^{j}, P^{j^{\prime}}\right)$. Relatively large differences between pairs of Pareto sets can be observed. The absolute value of this indicator has an implication as well: on average, the fraction of Pareto solutions in which a certain decision variable is Pareto optimal, differs $8.7 \%$. This can be considered as substantial, taking into account that some decision variables are (almost) always equal to 0 , or (almost) always nonzero (see table 5). Furthermore, also in this indicator there is a small difference between the average value within groups of runs $(8.4 \%)$ and between groups of runs (8.8\%). These differences seem too small to be substantial.

\subsection{Comparing Multiple Pareto Sets}

Finally, Figure 6 shows the attainment plots of the 5 identical parameter settings, for the objective combinations of total travel time and $\mathrm{CO}_{2}$ emissions resp. urban space used and operating benefit. For the first combination of objectives, some clear variation in results can be observed, mainly in the extreme ends of the Pareto set. This could already be seen in Figure 5. The second combination of objectives only shows a small boundary of uncertainty, also because the tradeoff region of the Pareto set is much bigger with these objectives.

\section{Conclusions}

In this paper the NSGA-II algorithm is applied to the multimodal transportation network design problem, as an example application of a multi-objective evolutionary algorithm. The performance of several runs of the optimization process is illustrated by a case study. The differences between multiple random outcomes of the algorithm are analyzed, both with a single parameter setting and with different parameter settings.

For this analysis, various indicators and methods are used to assess both the differences between the attained objective values as well as the differences between decision variables of the Pareto sets. Especially the newly defined indicators to compare Pareto sets in the decision space appeared to be useful to provide insight in the structure of the Pareto sets. 

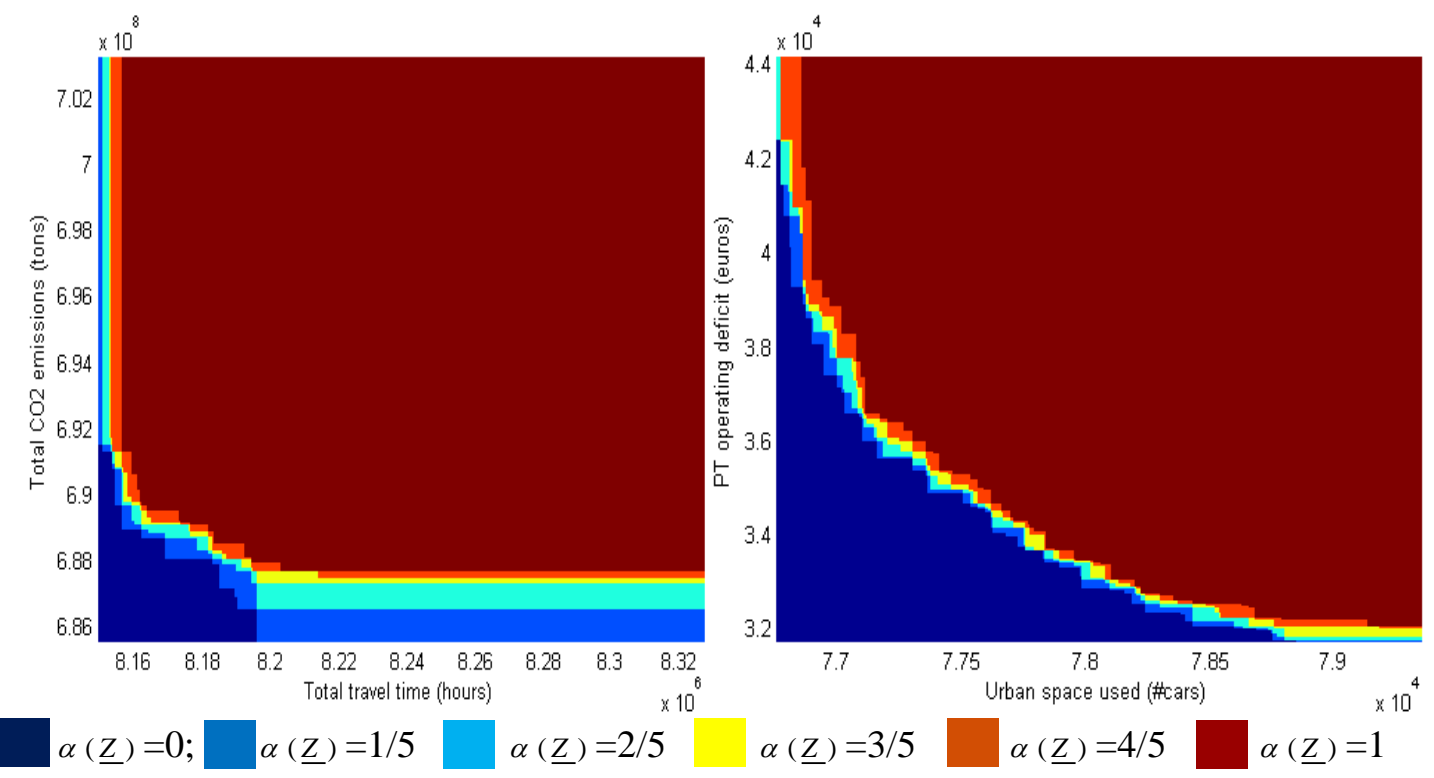

Figure 6. The Attainment Plots of the Outcome of Optimization Processes 1-5. The Blue Surface is attained by none of the Pareto Sets, while the Dark Red Surface is attained by all Pareto Sets

The first conclusion from this analysis is that the differences in the attained objective values are not too big among the runs: neither within runs with the same parameters nor between runs with different parameter settings. However, the mutation rate seems to be of influence on the result, so it seems wise to try at least a few different values for this parameter when setting up a new case study.

Secondly, based on the average distance to the nearest solution in the decision space and difference between the fractions of nonzero decision variables in two different Pareto sets, it can be concluded that the decisions to be taken are substantially different in all analyzed pairs of Pareto sets, so the advised decisions are influenced by the randomness of the algorithm. When taking decisions based on Pareto sets that are constructed by such an algorithm, more knowledge is needed on the sensitivity of decision variables with respect to the objective values, to identify important and less important decision variables.

Thirdly, variations caused by different parameters in the NSGA-II algorithm are slightly higher than the variations caused by randomness within the algorithm, based on several methods for comparison of Pareto sets. However, because this difference is too small to be substantial, we conclude that the influence of the parameter values to be chosen in the algorithm is not big, implying that the risk for running the algorithm with bad parameter values is small.

\section{Acknowledgements}

We thank The Netherlands Organisation for Scientific Research (NWO) for funding this research in the program Sustainable Accessibility of the Randstad (SAR): this research is part of the project Strategy towards Sustainable and Reliable Multimodal Transport in the Randstad (SRMT). Furthermore, we thank the City Region of Amsterdam for the use of the Venom data. 


\section{References}

[1] C. Coello, S. de Computación and C. Zacatenco, "Twenty years of evolutionary multi-objective optimization: A historical view of the field", IEEE Computational Intelligence Magazine, vol. 1, no. 1, (2006).

[2] L. J. J. Wismans, E. C. van Berkum and M. C. J. Bliemer, "Comparison of Multiobjective Evolutionary Algorithms for Optimization of Externalities by Using Dynamic Traffic Management Measures", Transportation Research Record: Journal of the Transportation Research Board, vol. 2263, (2011).

[3] V. Guihaire and J. K. Hao, "Transit network design and scheduling: A global review", Transportation Research Part A: Policy and Practice, vol. 42, no. 10, (2008).

[4] H. Yang and M. Bell, "Models and algorithms for road network design: a review and some new developments", Transport Reviews, vol. 18, no. 3, (1998).

[5] E. Miandoabchi, R. Farahani, W. Dullaert and W. Szeto, "Hybrid Evolutionary Metaheuristics for Concurrent Multi-Objective Design of Urban Road and Public Transit Networks", Networks and Spatial Economics, vol. 12, no. 3, (2012).

[6] Y. Hamdouch, M. Florian, D. Hearn and S. Lawphongpanich, "Congestion pricing for multi-modal transportation systems", Transportation Research Part B: Methodological, vol. 41, no. 3, (2007).

[7] A. Chen, J. Kim, S. Lee and Y. Kim, "Stochastic multi-objective models for network design problem", Expert Systems with Applications, vol. 37, no. 2, (2010).

[8] S. Sharma, S. Ukkusuri and T. Mathew, "Pareto Optimal Multiobjective Optimization for Robust Transportation Network Design Problem", Transportation Research Record: Journal of the Transportation Research Board 2090, (2009).

[9] G. Cantarella and A. Vitetta, "The multi-criteria road network design problem in an urban area", Transportation, vol. 33, no. 6, (2006).

[10] Y. Yin and S. Lawphongpanich, "Internalizing emission externality on road networks", Transportation Research Part D: Transport and Environment, vol. 11, no. 4, (2006).

[11] A. Sumalee, S. Shepherd and A. May, "Road user charging design: dealing with multi-objectives and constraints", Transportation, vol. 36, no. 2, (2009).

[12] B. Beltran, S. Carrese, E. Cipriani and M. Petrelli, "Transit network design with allocation of green vehicles: A genetic algorithm approach", Transportation Research Part C: Emerging Technologies, vol. 17, no. 5, (2009).

[13] P. Chakroborty, "Genetic Algorithms for Optimal Urban Transit Network Design", Computer-Aided Civil and Infrastructure Engineering, vol. 18, no. 3, (2003).

[14] E. Cipriani, S. Gori and M. Petrelli, "Transit network design: A procedure and an application to a large urban area", Transportation Research Part C: Emerging Technologies, vol. 20, no. 1, (2012).

[15] W. Fan and R. Machemehl, "Optimal Transit Route Network Design Problem with Variable Transit Demand: Genetic Algorithm Approach", Journal of Transportation Engineering, vol. 132, no. 1, (2006).

[16] M. Gallo, B. Montella and L. D'Acierno, "The transit network design problem with elastic demand and internalisation of external costs: An application to rail frequency optimisation", Transportation Research Part C: Emerging Technologies, vol. 19, no. 6, (2011).

[17] A. Mauttone and M. Urquhart, "A multi-objective metaheuristic approach for the transit network design problem", Public Transport, vol. 1, no. 4, (2009).

[18] L. dell'Olio, J. Moura and A. Ibeas, "Bi-Level Mathematical Programming Model for Locating Bus Stops and Optimizing Frequencies", Transportation Research Record: Journal of the Transportation Research Board 1971, (2006).

[19] S. Tahmasseby, "Reliability in urban public transport network assessment and design", TU Delft, (2009).

[20] F. Viti, S. F. Catalano, M. Li, C. Lindveld and H. J. van Zuylen, "Optimization Problem with Dynamic Route-Departure Time Choice and Pricing", 82nd Annual Meeting of the Transportation Research Board, Washington, D.C., (2003) January 12-16.

[21] Y. Sheffi, "Urban transportation networks: Equilibrium analysis with mathematical programming methods", Prentice-Hall, Englewood Cliffs, New Jersey, (1985).

[22] Z. Gao, J. Wu and H. Sun, "Solution algorithm for the bi-level discrete network design problem", Transportation Research Part B: Methodological, vol. 39, no. 6, (2005).

[23] K. Deb, "Multi objective Optimization Using Evolutionary Algorithms", John Wiley \& Sons, Chichester, UK (2001).

[24] K. Deb, A. Pratap, S. Agarwal and T. Meyarivan, "A fast and elitist multiobjective genetic algorithm: NSGA-II", IEEE transactions on evolutionary computation, vol. 6, no. 2, (2002).

[25] E. Zitzler, J. Knowles and L. Thiele, "Quality Assessment of Pareto Set Approximations, in Multiobjective Optimization", Edited J. Branke, K. Deb, K. Miettinen and R. Słowiński, Springer Berlin Heidelberg, (2008).

[26] L. While, P. Hingston, L. Barone and S. Huband, "A faster algorithm for calculating hypervolume, Evolutionary Computation", IEEE Transactions, vol. 10, no. 1, (2006). 
[27] E. Zitzler and L. Thiele, "Multiobjective evolutionary algorithms: A comparative case study and the strength pareto approach", IEEE transactions on evolutionary computation, no. 3, no. 4, (1999).

[28] J. Luttik, "The value of trees, water and open space as reflected by house prices in the Netherlands", Landscape and Urban Planning, vol. 48, no. 3-4, (2000).

[29] INFRAS, "Artemis: Assessment and reliability of transport emission models and inventory systems", Road Emission Model - Model Description. Workpackage 1100. Deliverable, no. 13, INFRAS, (2007).

\section{Authors}

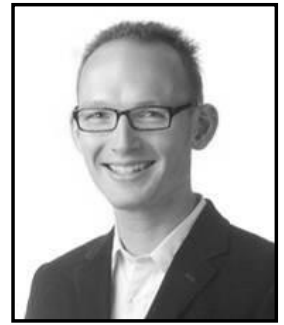

Ties Brands, is a $\mathrm{PhD}$ candidate at the Centre for Transport studies at the University of Twente (The Netherlands). He received his BSc degree (Civil Engineering) in 2004 and his two MSc degrees (Civil Engineering and Applied Mathematics) in 2008. His MSc thesis was on optimization of dynamic road pricing and was conducted at the Dutch consultancy company Goudappel Coffeng. After graduation, Ties started working at Goudappel Coffeng as a public transport consultant, with specialization in public transport modeling and data analysis. Parallel to his work as a consultant, he started a $\mathrm{PhD}$ project at the Centre for Transport Studies on multi-objective optimization of multimodal passenger transportation networks.

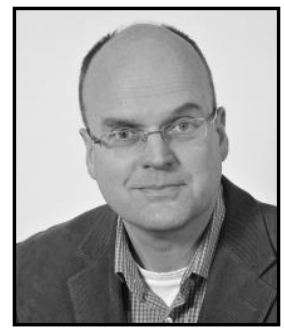

Eric van Berkum in the early 80's Eric van Berkum received his BSc and MSc in Applied Mathematics at the University of Twente (The Netherlands). After a relatively short career as a software engineer at Cap Gemini he moved in 1988 to Goudappel Coffeng, a traffic and transport consultancy company in Deventer. He worked there for 21 years in which he has had several positions in this company. His main interest has been on transport modeling, networks, travel behaviour and traffic management. In 1998 he became a parttime professor in traffic management at the Centre for Transport Studies. In 2009 he left Goudappel Coffeng and became a full professor and also head of the Centre for Transport Studies at the University of Twente. 